\title{
La génétique du cancer colorectal
}

Au moins quatre loci génétiques seraient impliqués dans la survenue des cancers colorectaux. Dans les formes familiales, un gène associé à la polypose rectocolique est localisé sur le chromosome 5 et un autre gène prédisposant au cancer colorectal sans polypose est situé sur le chromosome 18. La connaissance de ces localisations devrait rapidement permettre de reconnaître précocement les sujets prédisposés à ce type de cancers. Dans les cas sporadiques, l'analyse cytogénétique et les méthodes de génétique moléculaire montrent la perte très fréquente d'un chromosome 18 et du bras court d'un chromosome 17. Parmi les anomalies les moins fréquentes, la perte d'un fragment du bras long du chromosome 5 et la mutation ponctuelle de l'oncogène $K \boldsymbol{K}$ ras sur le chromosome 12 sont chacune observées dans approximativement un tiers des cas. L'apparition des adénocarcinomes est très souvent précédée de poly-adénomes, véritables proliférations monoclonales précancéreuses. Au cours de la progression tumorale, l'ordre de survenue des anomalies cytogénétiques peut en général être précisé. L'hypothèse de l'intervention d'antioncogènes dans la carcinogenèse colorectale est discutée.

\section{Gilles Thomas \\ Chargé de recherche au Cnrs}

\section{Martine Muleris \\ Chargée de recherche au Cnrs}

\section{Rémy-Jacques Salmon} Chef de service en chirurgie générale

\section{ADRESSES}

G. Thomas, M. Muleris : Institut Curie, section de biologie, 26, rue d'Ulm, 75231 Paris Cedex 05, France.

R.-J. Salmon: Institut Curie, section médicale et hospitalière, 26, rue d'Ulm, 75231 Paris es cancers colorectaux sont, tous sexes confondus, les cancers les plus fréquents dans les pays occidentaux. Ils entraînent une mortalité dans $50 \%$ des cas au cours des cinq années qui suivent leur diagnostic ; ils constituent de ce fait la deuxième cause de mortalité par cancer, immédiatement après le cancer du poumon. Comme pour tous les cancers digestifs, de nombreuses études épidémiologiques ont cherché à évaluer le rôle de l'alimentation sans avoir jusqu'à présent débouché sur des stratégies possibles de prévention*. En ce qui concerne le mécanisme de carcinogenèse, il était admis que la majorité des facteurs de risque agit par l'intermédiaire d'agents génotoxiques provo-

\footnotetext{
* Voir sur ce point l'article du docteur Monique Martin, La cancérogenèse colique humaine et expérimentale, dans ce numéro, page 281 .
}

quant des altérations dans le génome des cellules de l'épithélium rectocolique. Or, la nature de certaines de ces anomalies vient d'être reconnue. De plus, récemment, des gènes prédisposant à la survenue de ces cancers et transmis héréditairement dans certaines familles ont été localisés sur la carte génétique humaine. A la lumière de ces découvertes, il est aujourd'hui intéressant de s'interroger sur les relations pouvant exister entre les mutations observées dans les cancers héréditaires et les altérations acquises impliquées dans les cancers colorectaux sporadiques.

\section{Les prédispositions héréditaires}

On estime que 5 à $10 \%$ des cancers colorectaux sont héréditaires. Les prédispositions aux adénocarcinomes rectocoliques qui ont pu être étudiées très précisément révèlent une transmission autosomique 


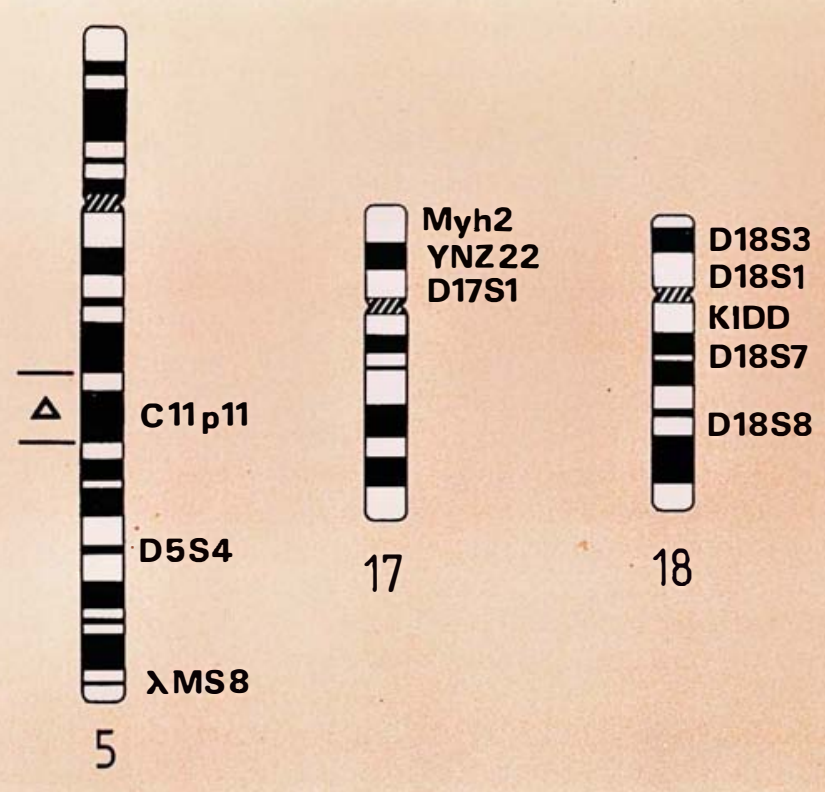

Figure 1. Délétions chromosomiques des cancers sporadiques et gènes impliqués dans les formes familiales. Le chromosome 5 dont la délétion

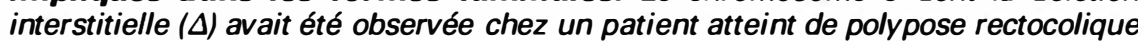
porte le gène FAP localisé au voisinage de la sonde C11p11. Dans les cas sporadiques, l'utilisation des sondes D5S4 et MS8 a permis de noter des pertes alléliques dans approximativement un tiers des cas. Sur le chromosome 18 le groupe sanguin Kidd est associé étroitement à un gène impliqué dans les cancers familiaux du groupe Lynch II. Les sondes RFLPs étagées sur toute la longueur de ce chromosome montrent des pertes alléliques fréquentes dans les formes sporadiques. Dans ces mêmes formes sur le bras court du chromosome 17 les pertes alléliques sont très souvent observées avec les sondes D17S1, YNZ22 et Myh2. A l'heure actuelle la co-ségrégation d'un gène de cette région avec une prédisposition familiale au cancer n'est pas connue.

dominante. Il est schématiquement possible de distinguer deux grands groupes de syndromes familiaux. Le premier rassemble les familles dont les membres malades sont porteurs d'une polypose rectocolique. Ces patients sont aisément reconnus car ils ont tous des poly-adénomes multiples, véritables marqueurs cliniques de la maladie. Le deuxième groupe est constitué des familles dans lesquelles les cancers colorectaux héréditaires ne sont pas associés à la présence de poly-adénomes. Bien qu'il n'y ait pas dans ce cas de marqueur clinique aisément reconnaissable, on estime que ce groupe est cinq fois plus grand que le précédent. Cette distinction suivant la présence ou non de poly-adénomes sem$\mathrm{m} / \mathrm{s} n^{\circ} 5$ vol. 4, mai 88 aboutiraient dans un avenir proche. Cependant la découverte, chez un patient sans antécédent familial particulier, d'un retard mental associé à tous les signes cliniques de la polypose rectocolique familiale a très certainement permis de gagner de nom- breux mois pour la localisation du gène FAP (familial adenomatous polyposis). En effet l'analyse cytogénétique des cellules sanguines de ce patient devait révéler une petite délétion interstitielle du bras long du chromosome 5 dont les points de cassures étaient cependant difficiles à préciser [1](figure 1). Étant donnée la présence fréquente de microdélétions chez les malades atteints de retard mental, cette observation passa quasiment inaperçue. Ce fut le grand mérite de W.F. Bodmer d'avoir envisagé la possibilité que la délétion observée sur le chromosome 5 puisse être aussi responsable de l'apparition de la polypose, et d'avoir recherché une liaison génétique entre la maladie et des sondes spécifiques du chromosome 5. Une chance extraordinaire voulut que ce groupe utilisât la sonde RFLP* Cllpll localisée en 5 q2l pour mettre en évidence cette liaison. Cette sonde peu polymorphe se révéla en effet extrêmement proche du locus FAP puisqu'actuellement il n'a pas été possible d'observer la ségrégation des deux caractères $[2,3]$. De manière très intéressante, l'étude de co-ségrégation menée sur des familles atteintes d'une variante de la polypose rectocolique familiale, le syndrome de Gardner, semble montrer une localisation identique du gène sur le chromosome 5 . Il est donc vraisemblable que, comme dans le cas des myopathies de Becker et de Duchenne, cette variante soit provoquée par une mutation différente du même gène.

Les prédispositions aux adénocarcinomes rectocoliques non accompagnés de polypose ont fait l'objet d'une recherche systématique de coségrégation avec différents marqueurs protéiques sériques. Lynch avait trouvé, il y a déjà plusieurs années, une liaison génétique probable avec le groupe sanguin Kidd [4]. Dans les familles étudiées, lorsqu'il séparait les prédispositions familiales strictement limitées au côlon (Lynch I) de celles se traduisant par de nombreux cancers extra-digestifs, en particulier gynécologiques
* RFLP: restriction fragment length polymorphism, polymorphisme de taille des fragments de restriction $\left(\mathrm{m} / \mathrm{s}\right.$, suppl. au $n^{\circ} 7$, vol. 3, p. 4). 


\section{RÉFÉRENCES}

1. Herrera L, Kakati S, Gibas L, et al. Gardner syndrome in a man with an interstitial deletion of 5q. Am J Med Genet 1986; 25 : 473-6.

2. Bodmer WF, Bailey CJ, Bodmer J, et al. Localization of the gene for familial adenomatous polyposis on chromosome 5 . Nature 1987 ; 328 : 614-9.

3. Leppert M, Dobbs M, Scambler P, O'Connell $\mathrm{P}$, et al. The gene for familial polyposis coli maps to the long arm of chromosome 5 . Science 1987 ; 238 : 1411-3.

4. Lynch HT, Schvelke GS, Kimberling WJ, et al. Hereditary nonpolyposis colorectal cancer (Lynch Syndromes I and II). Cancer 1985 ; $56: 939-51$.

5. Bos JL, Fearon ER, Hamilton SR, et al. Prevalence of ras gene mutations in human colorectal cancers. Nature 1987 ; 327 : 293-7.

6. Forrester K, Almoguera C, Han K, et al. Detection of high incidence of $\mathrm{K}$-ras oncogenes during human colon tumorigenesis. Nature 1987 ; 327 : 298-303.

7. Muleris M, Salmon RJ, Dutrillaux AM, et al. Characteristic chromosomal imbalances in 18 near-diploid colorectal tumors. Cancer Genet Cytogenet 1987 ; 29 : 289-302.

8. Fearon ER, Hamilton SR, Vogelstein B. Clonal analysis of human colorectal tumors Science 1987 ; 238 : 193-6.

9. Monpezat JPh, Delattre O, Bernard A, et al. Loss of alleles on chromosome 18 and on the short arm of chromosome 17 in polyploid colorectal carcinomas. Int J Cancer 1988 ; 41 . 404-8.

10. Solomon E, Voss R, Hall V, et al. Chromosome 5 allele loss in human colorecta carcinomas. Nature $1987 ; 328: 616-9$.

11. Muleris M, Salmon RJ, Dutrillaux B. Existence of 2 dist inct processes of chromosomal evolution in near-diploid colorectal tumors. Cancer Genet Cytogenet 1988 (sous
(Lynch II), il faisait apparaître une liaison significative entre le groupe sanguin Kidd et le gène prédisposant au Lynch II. Au moment de cette découverte, le groupe sanguin Kidd n'avait pas été localisé sur la carte génétique humaine, si bien qu'il était difficile d'approfondir ces résultats avec des techniques de génétique moléculaire. Au dernier congrès sur la carte génétique de l'homme, tenu à Paris en septembre 1987, plusieurs groupes ont démontré une localisation du facteur Kidd dans une région proche du centromère du chromosome 18. Il est donc plausible que, dans un avenir proche, les résultats obtenus par Lynch seront étendus et précisés en utilisant des sondes RFLPs.

Bien que seulement 5 à $10 \%$ des adénocarcinomes rectocoliques taires, l'étude épidémiologique montre qu'un individu dont un parent au premier degré a présenté un adénocarcinome rectocolique a deux fois plus de risques de développer une soient considérés comme hérédi-

tumeur du même type. Cette remarque suggère la fréquente intervention, même dans les cas apparemment sporadiques, de déterminismes génétiques à faible pénétrance encore mal identifiés. Une recherche systématique du type de celle réalisée pour la polypose rectocolique familiale est ici difficilement envisageable. On peut cependant examiner si un gène polymorphe particulier peut être impliqué dans une prédisposition individuelle en comparant la distribution des allèles dans la population atteinte et dans une population témoin. Il faut savoir que cette approche, à la différence de celle réalisée sur les familles, n'est pas dénuée de difficultés d'interprétation importantes. Deux loci ont fait l'objet de recherches intensives : le système HLA et l'oncogène $\mathrm{Ha}$ ras. A ce jour il n'a pas été montré dans la maladie qui nous intéresse de différence significative dans la distribution des allèles entre groupe témoin et groupe malade. Cependant on a pu montrer une liaison du locus

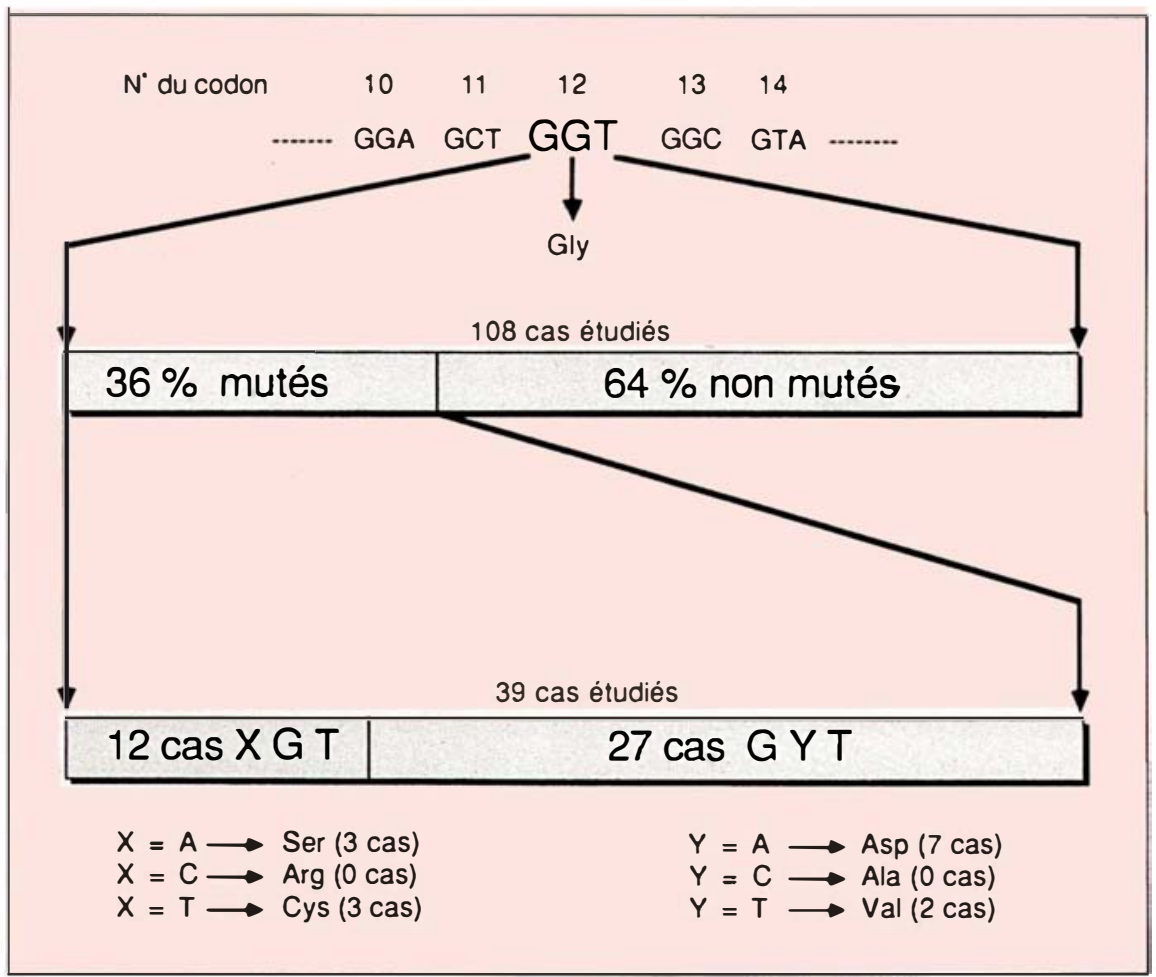

Figure 2. La mutation du codon 12 de Ki-ras dans le cancer colorectal. Dans un tiers des cancers colorectaux l'oncogène Ki-ras est muté sur le codon 12. Seuls des changements sur la première ou la deuxième lettre de ce codon affectent la structure de la protéine. La deuxième lettre est deux fois plus souvent impliquée dans les mutations détectées. On constate que la transversion $G$ vers $A$ est plus fréquente que les transitions $G$ vers $T$ ou $G$ vers $C$. 


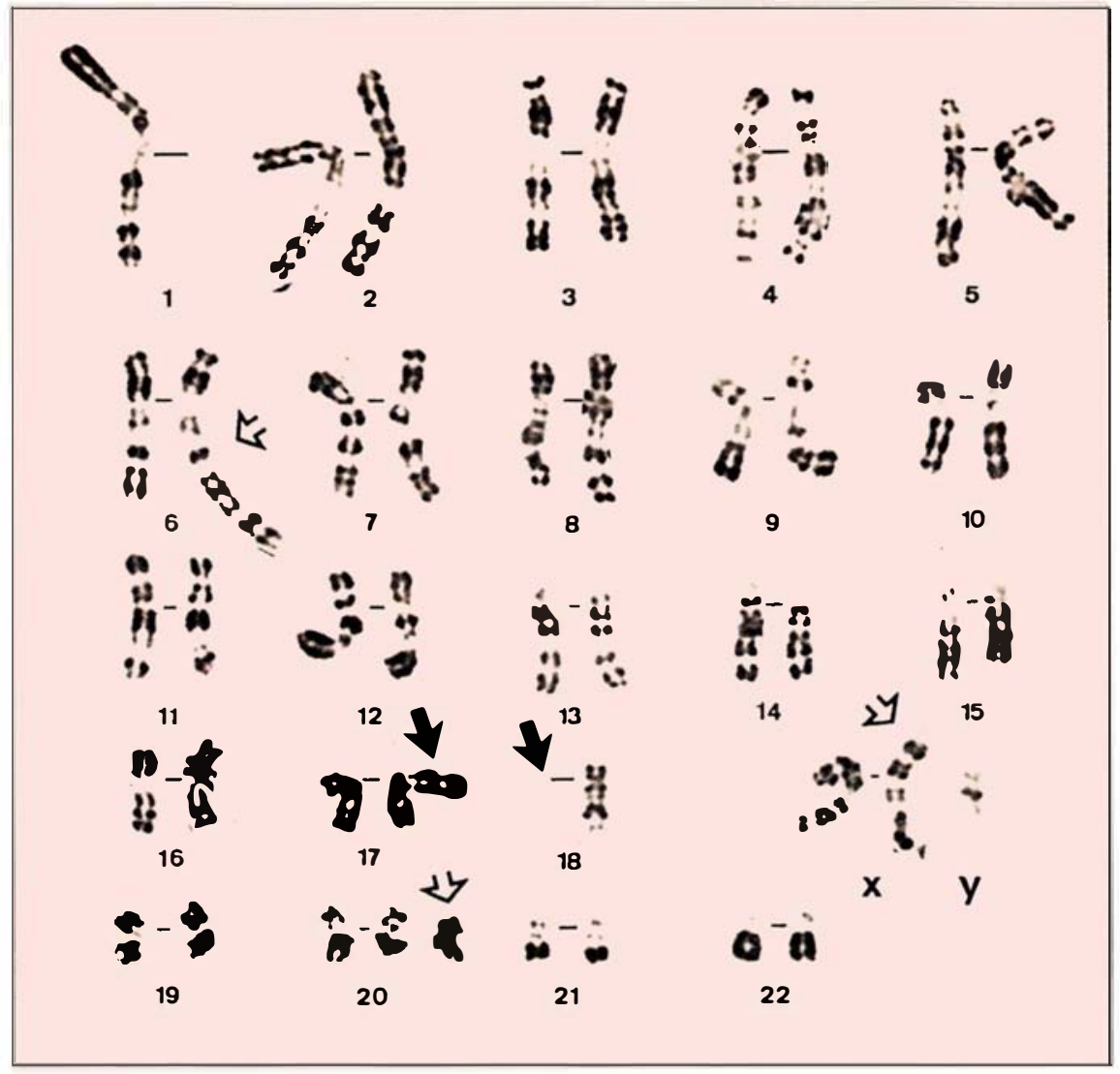

Figure 3. Exemple de caryotype de tumeur colorectale. Caryotype caractéristique d'une cellule de cancer colorectal sporadique: le manque d'un chromosome 18 et le remaniement d'un chromosome 17 aboutissant à la perte de son bras court sont les anomalies les plus fréquentes (flèches noires). Les autres modifications chromosomiques - remaniement d'un chromosome 1 (ici translocation avec le 6) aboutissant à la perte du bras court, gain d'un chromosome 20 et d'un chromosome $X$ - qui sont observées dans toutes les cellules tumorales de ce patient, sont moins fréquemment retrouvées dans d'autres cancers colorectaux (flèches blanches). Le chromosome 5 apparaît ici normal.

HLA avec des maladies inflammatoires du côlon et du rectum (maladie de Crohn, rectocolite hémorragique) et à l'intolérance au gluten, maladies où le risque de développement de cancer colorectal au cours de l'évolution est connu.

\section{Les anomalies génétiques acquises}

Il y a déjà plusieurs années l'étude par transfection d'ADN extrait de cellules cancéreuses dans des cellules indicatrices NIH 3T3 a permis de montrer la présence d'oncogènes $\mathrm{m} / \mathrm{s} n^{\circ} 5 \mathrm{vol} .4$, mai 88 sont détectées dans près de deux cinquièmes des cas étudiés $[5,6]$. L'activation est rarement due à une mutation sur le codon 61. Dans la très grande majorité des cas c'est le codon 12, de séquence GGT, qui est affecté (figure 2). L'étude de la répartition des mutations trouvées sur ce dernier codon est intéressante. Le premier $\mathrm{G}$ n'est muté que dans un tiers des cas, le deuxième $G$ étant muté dans tous les autres cas. Une mutation sur la troisième lettre n'affecte pas la structure de la protéine et n'a pas été observée. Alors que la mutation $G$ vers $C$ n'a pas encore été trouvée dans les cancers rectocoliques (elle a été détectée pour des cancers ayant d'autres localisations) et semble donc rare, les mutations $G$ vers $T$ et surtout $G$ vers $A$ sont les plus fréquentes. Ces observations constituent l'une des toutes premières informations précises concernant la mutagenèse ponctuelle sur un locus déterminé dans l'espèce humaine. $\mathrm{La}$ fréquence des mutations $\mathrm{Ki}$-ras suggère qu'elles participent à l'un des mécanismes génétiques préférentiels présidant au développement des adénocarcinomes rectocoliques. Il peut être proposé que, pour les trois cinquièmes des cas ne semblant pas présenter cette activation, une altération génétique fonctionnellement équivalente dans la carcinogenèse colique à celle de Ki-ras aurait pu survenir. Il est de ce point de vue intéressant de noter la découverte récente chez l'homme de plusieurs autres membres de la famille ras.

Il faut souligner que le caryotype des cellules carcinomateuses coliques présente des aberrations chromosomiques caractéristiques. Celles-ci ne sont pas, comme dans les cas bien connus du lymphome de Burkitt et de la leucémie myéloïde chronique, caractérisées par des points de cassure chromosomiques précis mais plutôt par des gains et surtout des pertes de fragments chromosomiques ou même de chromosomes entiers [7] (figure 3). Les anomalies les plus fréquentes sont la perte du bras court d'un chromosome 17 et de la totalité d'un chromosome 18 , trouvées respectivement dans $90 \%$ et $80 \%$ des cas. La région du chromosome 5 portant le gène de la polypose rectocolique familiale est trouvée délétée dans un quart des cas. 
Actuellement, pour des raisons techniques, seulement la moitié des adénocarcinomes rectocoliques sporadiques peuvent être étudiés en cytogénétique. Il est cependant possible, par l'utilisation de sondes RFLP, d'étendre les conclusions de ces études à des tumeurs dont le caryotype n'a pas été déterminé. En effet, en étudiant des individus constitutionnellement hétérozygotes pour des sondes portées par les segments chromosomiques trouvés habituellement délétés dans les tumeurs, il est possible de rechercher si, à la différence des cellules normales, un des allèles est perdu dans toutes les cellules cancéreuses. De telles pertes alléliques ont été trouvées dans $75 \%$ des cas pour des sondes portées par le bras court du chromosome 17 et dans $80 \%$ des cas lorsque ces sondes sont spécifiques du chromosome $18[8,9]$ (figure 4). Cette technique montre aussi que dans un tiers des cas un fragment du chromosome 5 a été perdu [10]. Ces résultats sont donc en complet accord avec ceux obtenus par la cytogénétique. Non seulement ils mettent en évidence les délétions mais encore démontrent que les cellules cancéreuses proviennent toutes d'une seule cellule ancestrale dans laquelle à l'origine se sont produites des pertes alléliques.

\section{Le poly-adénome : tumeur monoclonale pré-cancéreuse}

L'origine mono- ou polyclonale du poly-adénome fait depuis longtemps l'objet de controverses. Cependant, grâce en particulier à une technique d'échantillonnage très précise, l'ambiguité est aujourd'hui levée: les poly-adénomes coliques sont constitués d'une population cellulaire dérivant d'une seule cellule de la muqueuse colique. La démonstration a été réalisée chez les femmes portant certaines hétérozygoties sur le chromosome X [8] (figure 5). Il est en effet possible chez ces femmes de distinguer le chromosome $\mathrm{X}$ provenant du père de celui provenant de la mère. On sait aussi que chaque cellule inactive un des deux chromosomes $\mathrm{X}$; cette inactivation est

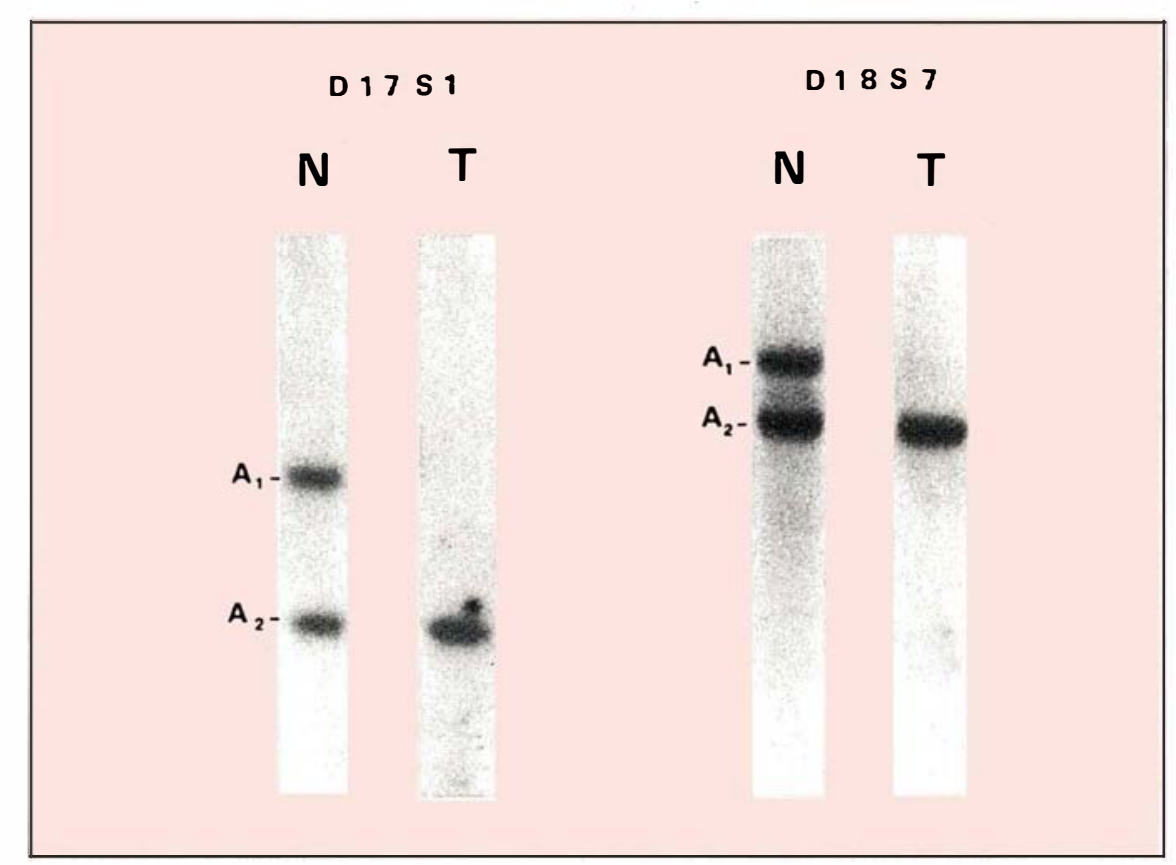

Figure 4. Exemple de pertes alléliques dans une tumeur rectocolique. La comparaison des profils obtenus par la technique de Southern pour I'ADN normal (N) et l'ADN tumoral (T) d'un même patient fait ressortir l'absence simultanée dans I'ADN tumoral d'un allèle porté par le bras court du chromosome 17 (D17S1) et d'un allèle porté par le chromosome 18 (D18S7).

culières, de méthylations qui inhibent l'action de certaines enzymes de restriction sur l'ADN de ce chromosome. Sauf exception, cette inactivation se fait au hasard, et à un stade assez tardif du développement, si bien que chaque organe est constitué approximativement d'un nombre égal de cellules ayant inactivé le chromosome $\mathrm{X}$ soit paternel, soit maternel. Une fois établie, l'inactivation d'un chromosome $\mathrm{X}$ semble pratiquement irréversible. En particulier après une mitose, le même chromosome $\mathrm{X}$ est inactivé dans les deux cellules filles résultantes. On a pu ainsi montrer que toutes les cellules d'un poly-adénome colique ont inactivé le même chromosome $\mathrm{X}$ et donc proviennent toutes d'une même cellule.

Les poly-adénomes colorectaux peuvent être considérés comme des lésions pré-cancéreuses. Cette notion résulte essentiellement du fait que, dans 60 à $80 \%$ des cancers, le carcinome semble dériver d'un poly-adénome. Il a été possible d'étudier conjointement le génome de l'adénocarcinome et celui de l'adénome semblant lui avoir donné naissance $[5,6]$.
A plusieurs reprises des cellules portant la mutation $K i$-ras ont été retrouvées dans la partie adénomateuse suggérant fortement que la mutation Ki-ras préexiste dans le poly-adénome et qu'une ou plusieurs altérations génétiques supplémentaires seraient nécessaires pour passer du stade adénome au stade carcinome. La perte du bras court du chromosome 17 pourrait bien être une de ces étapes puisque sur 30 cas étudiés, elle n'a été trouvée qu'une fois dans un adénome qui présentait cependant un caractère fortement dysplasique pouvant s'apparenter à celui d'un carcinome in situ.

\section{La progression tumorale}

Une tumeur cancéreuse est un processus dynamique en perpétuelle évolution. Si l'instabilité génétique des cellules cancéreuses est généralement considérée comme une simple conséquence de la cancérisation, elle peut en outre apparaître comme un facteur important leur permettant de plus grandes possibilités d'adaptation à un mode de fonctionnement imprévu. La sélection par les 


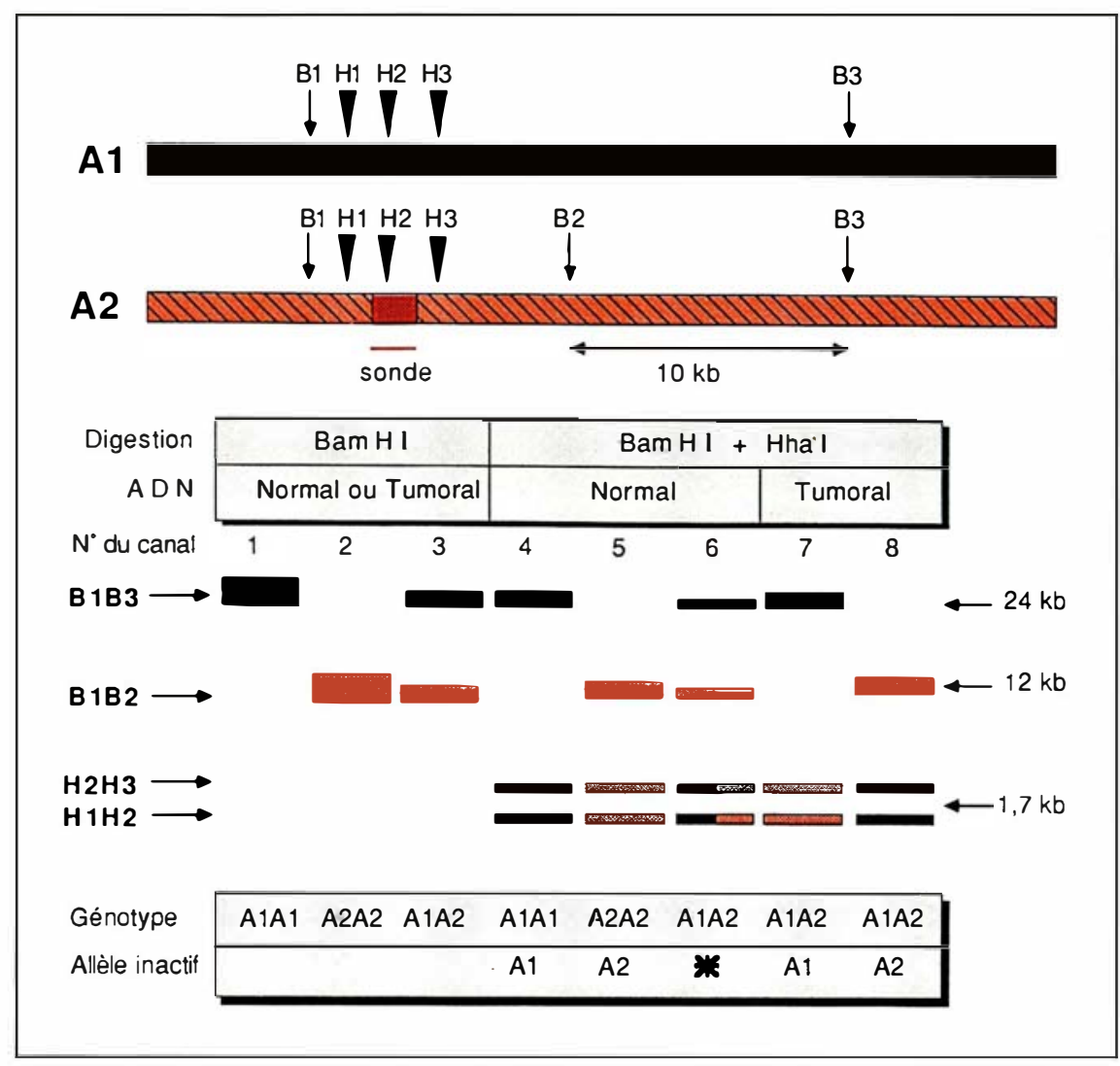

Figure 5. Démonstration de la monoclonalité d'une prolifération tumo-

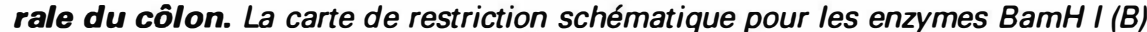
et Hha I $(H)$ est présentée pour les allèles A1 et A2 du gène HPRT (hypoxanthineguanine phosphoribosyltransférase) portés par le chromosome $X$. Ces allèles ne diffèrent que par le site de restriction B2. L'analyse par la technique de Southern après une digestion par l'enzyme BamH l et l'utilisation de la sonde indiquée permettent de distinguer les femmes homozygotes (canaux 1 et 2) des femmes hétérozygotes (canal 3). L'enzyme Hha I ne peut pas couper les sites H1, H2, H3 qui sont méthylés sur le chromosome $X$ inactif. L'enzyme coupe ces mêmes sites non méthylés sur le chromosome $X$ actif. La double digestion BamH I + Hha I démontre pour de l'ADN provenant de tissu normal chez les femmes hétérozygotes que la moitié des allèles A1 et la moitié des allèles A2 sont portées par le chromosome $X$ inactif ( $*$, canal 6). En revanche, chez les femmes hétérozygotes, dans I'ADN d'une tumeur bénigne ou cancéreuse du côlon la disparition de la bande B1B2 (canal 7) ou B1B3 (canal 8) établit qu'un des allèles est entièrement porté par le chromosome $X$ actif. Cette observation montre que la prolifération tumorale provient d'une seule cellule qui, à travers la succession des mitoses, transmet l'inactivation particulière de son allèle à l'ensemble des cellules tumorales. L'allèle A1 et les fragments de restriction qui en dérivent sont représentés en noir. L'allèle A2 et les fragments de restriction en dérivant sont représentés en rose avec une trame hachurée. Dans le canal 6, les fragments H1 H2 et H2H3 dérivant des deux allèles sont représentés avec les deux symboles de couleur (noir et rose tramé). $k b=$ kilobases

contraintes du milieu des cellules les mieux adaptées provoque une véritable évolution génétique qui conduit leur a donné naissance.

Une partie de cette évolution est perceptible dans l'analyse cytogénétique [11]. En effet, dans une tumeur du côlon donnée, une hétérogénéité caryotypique est souvent observée. Bien que toutes les cellules caryotypées aient en commun plusieurs anomalies, certaines cellules possèdent par rapport à cette formule chromosomique de base, des anomalies surajoutées. On observe ainsi, au sein de la même tumeur, la présence de cellules avec différentes formules caryotypiques qui dérivent les unes des autres, et qui permettent donc de préciser l'ordre de survenue des différentes anomalies.

Cependant, cette analyse ne permet pas de déterminer la première aberration chromosomique apparue, puisque toutes les cellules ont en commun non pas une seule mais plusieurs anomalies. Il est peu vraisemblable que ces dernières soient toutes apparues en même temps dans la cellule cancéreuse initiale. Il semble plutôt que, antérieurement à l'apparition de cette cellule, il existait un groupe de cellules ayant une forte instabilité génétique et commençant à échapper à l'homéostasie. Progressivement, les mutations ont pu s'accumuler jusqu'au jour où une combinaison appropriée de mutations au sein d'une même cellule lui a conféré un avantage sélectif, cette cellule prenant le pas sur toutes les autres et imposant sa descendance.

\section{Des anti-oncogènes dans la cancérogenèse rectocolique?}

Il est frappant de noter que, très généralement, les loci anormaux dans les familles prédisposées à certains cancers sont fréquemment trouvés délétés dans les cancers correspondants, que ceux-ci soient sporadiques ou rattachés à un syndrome familial.

Une tentative d'explication intégrant cette observation au caractère autosomique dominant de la transmission dans ces familles repose sur le concept d'anti-oncogène. Un anti-oncogène est un gène codant pour une fonction nécessaire permettant à la cellule d'obéir au contrôle, par l'organisme, de sa prolifération et de sa différenciation. Dans les cas sporadiques, un premier événement muta- 
tionnel inactiverait un seul des deux gènes présents dans la cellule. Cette inactivation ne se traduirait ni par des variations phénotypiques ni par des altérations génétiques qui puissent être repérées facilement par les techniques actuelles. Elle passerait inaperçue s'il ne survenait un second événement qui, faisant disparaître l'expression du gène resté jusqu'à présent normal, priverait totalement la cellule de cette fonction.

Dans la grande majorité des cas, cette étape surviendrait par perte du segment chromosomique portant le gène fonctionnel. Ce serait uniquement ce second événement qui serait observé en cytogénétique ou par l'étude des pertes alléliques. Chez les membres malades dans les familles prédisposées, la mutation initiale serait présente à l'état constitutionnel, si bien que seule, la deuxième étape deviendrait nécessaire pour permettre l'expression phénotypique du cancer. L'inactivation d'antioncogènes dans le mécanisme de la carcinogenèse est aujourd'hui solidement établie dans le cas du rétinoblastome. Elle semble très vraisemblable pour le néphroblastome et le méningiome dans leurs formes familiales et sporadiques. Cependant, la fréquence des pertes alléliques retrouvées dans les formes sporadiques de nombreux cancers très différents (poumon, sein, vessie, etc.) conduit à s'interroger, bien que les formes familiales correspondantes ne soient pas bien caractérisées, sur la généralité de l'intervention d'antioncogènes.

Dans le cas du cancer du côlon, nous n'avons pas d'argument particulier pour proposer que le bras court du chromosome 17 soit porteur d'un anti-oncogène. En revanche, nous venons de voir que deux loci prédisposant à des formes différentes de cancers colorectaux familiaux sont souvent trouvés délétés dans les formes sporadiques. Le gène FAP, localisé sur le chromosome 5 peut-il être considéré comme un anti-oncogène? Il existe un contraste entre les altérations cytogénétiques du chromosome 5, relativement peu fréquentes et parfois tardives dans la chronologie des événements mutationnels, et le rôle anti-oncogène devrait empêcher même à l'état hémizygote l'apparition de poly-adénome, étape précoce et habituelle des cancers sporadiques. Des résultats préliminaires suggèrent que dans les poly-adénomes les hétérozygoties portées par le chromosome 5 sont fréquemment conservées. Il semble donc que, dans ces cas, soit le gène FAP n'est pas atteint, soit sa mutation n'est pas strictement récessive. La perte secondaire d'un fragment chromosomique portant le gène FAP pourrait alors n'être qu'un des éléments de la progression tumorale, (le gène FAP restant n'étant d'ailleurs pas nécessairement muté). Dans une telle situation le gène FAP ne se comporterait donc pas comme un anti-oncogène stricto sensu.

Les pertes alléliques de loci portés par le chromosome 18 sont-elles révélatrices de l'existence d'un anti-oncogène sur ce chromosome qui serait atteint dans les familles se rattachant au groupe Lynch II ? La fréquence et la précocité de ces délétions dans les adénocarcinomes rectocoliques sont en faveur de cette hypothèse. Cependant, par définition ces familles sont prédisposées à des cancers extradigestifs. Si les cancers du groupe Lynch II n'ont pas encore fait l'objet d'études cytogénétiques, les cas sporadiques de même localisation (sein, utérus, ovaire) ne présentent pas de délétion récurrente du chromosome 18. Cette observation qui semble exclure un rôle fréquent du chromosomel 8 dans les cancers sporadiques de l'appareil gynécologique, n'élimine cependant pas l'hypothèse d'un anti-oncogène porté par ce chromosome et impliqué préférentiellement dans la carcinogenèse rectocolique.

Le travail des prochaines années permettra de mieux comprendre comment les différentes altérations décrites coopèrent pour déterminer l'apparition de ces tumeurs et leur évolutivité. Il est vraisemblable que des marqueurs génétiques auront bientôt leur place dans les stratégies de prévention et de dépistage. En attendant, les connaissances actuelles doivent, à brève échéance, permettre un conseil génétique pour de nombreuses familles prédisposées au cancer colorectal

\section{Summary}

The genetics of colorectal cancer has recently made important progresses. A gene associated to familial polyposis coli has been located on chromosome 5. An other gene implicated in predisposition to colorectal cancer without polyposis is located on chromosome 18 . These results will rapidly lead to the development of prenatal diagnosis for these diseases. In the sporadic cases the analysis by cytogenetic or molecular biology techniques demonstrates the very frequent losses of chromosome 18 or of the short arm of chromosome 17. Among the less frequent anomalies, the loss of a fragment of the long arm of chromosome 5 and the point mutation on the Ki-ras oncogene have been observed in approximately a third of the cases. Colorectal carcinogenesis is a multistep process. Frequently one step is an adenoma, a genuine precancerous monoclonal proliferation. In the course of tumor progression the order of occurrence of the cytogenetic anomalies can generally be found. The hypothesis of the involvement of antioncogenes in colorectal carcinogenesis is discussed.

\section{Remerciements}

Nous tenons à remercier tout particulièrement le docteur B. Dutrillaux dont le rôle a été primordial dans l'élaboration de plusieurs idées présentées dans cet article. Le travail des auteurs a bénéficié d'un soutien du comité de Paris de la Ligue Nationale contre le Cancer. Nous sommes également reconnaissants à Mlle Marie-France Malot pour l'aide qu'elle a apportée à la préparation de ce manuscrit et au docteur Anne Bernard pour ba relecture et la critique du texte.

\section{TIRÉS A PART}

G. Thomas : Institut Curie, section de biologie, 26, rue d'Ulm, 7523I Paris Cedex 05, France. 